\title{
Study of strongly correlated electrons in nanoscopic and bulk forms: some recent results
}

\author{
G Alvarez ${ }^{1}, \mathbf{K}$ A Al-Hassanieh ${ }^{2,3,4}$, F Popescu ${ }^{5}, \mathrm{C} \mathrm{SSen}^{4}, \mathrm{~A} \mathrm{Moreo}^{2,3}$ and \\ E Dagotto ${ }^{2,3}$ \\ ${ }^{1}$ Computer Science and Mathematics Division and Center for Nanophase Materials Sciences, \\ Oak Ridge National Laboratory, Oak Ridge, TN 37831, USA \\ ${ }^{2}$ Condensed Matter Sciences Division, Oak Ridge National Laboratory, Oak Ridge, TN 37831 , \\ USA \\ ${ }^{3}$ Department of Physics and Astronomy, University of Tennessee, Knoxville, TN 37996, USA \\ ${ }^{4}$ National High Magnetic Field Laboratory and Department of Physics, Florida State University, \\ Tallahassee, FL 32306, USA \\ ${ }^{5}$ Department of Physics, Florida State University, Tallahassee, FL 32306, USA
}

Received 15 August 2006, in final form 22 August 2006

Published 6 March 2007

Online at stacks.iop.org/JPhysCM/19/125213

\begin{abstract}
Recent progress in the numerical study of various strongly correlated electronic systems is reviewed. The study of transport in single molecule conductors and quantum dots is addressed with a recently proposed adaptive time-dependent density-matrix-renormalization group (DMRG). Experiments involving nonlocal spin control and their numerical simulation are also discussed. A section is devoted to recent efforts in the study of spin-fermion models for colossal magnetoresistive manganites, where we present insights on the effect of disorder and electron-phonon coupling. Finally, using a dynamical mean field approach, we review calculations in the area of diluted magnetic semiconductors that provides guidelines on how the Curie temperature could be increased in these itinerant ferromagnetic systems.
\end{abstract}

(Some figures in this article are in colour only in the electronic version)

\section{Introduction}

In several materials of technological interest the interactions between the electrons are collective, and these systems, referred to as strongly correlated electronic systems, display a broad range of interesting and important phenomena.

One example is the study of electron transport through single-molecule conductors and quantum dots. These nanostructures are not only candidates for novel potential applications, such as molecular electronics and quantum computing, but also provide well-controlled and tunable systems for studying interesting many-body effects. In this paper, we review recent results in this area, including the Coulomb blockade and Kondo effects in section 2. 
Another example is the colossal magnetoresistive (CMR) effect in manganites. In recent years, much progress has been made in this context after the realization that several competing states, with quite different properties, are very close in energy in these compounds. As a consequence, small variations in carrier density, temperature, pressure, magnetic fields, and other variables often lead to giant responses. Moreover, the effect of quenched disorder appears to be important in understanding the colossal magnetoresistive effect, according to recent investigations that we review in section 3. This combination of strong correlation and disorder effects, and the simultaneous relevance of several degrees of freedom such as spin, charge, orbital, and lattice, lead to a plethora of unusual properties, with tremendous potential for applications.

In addition, we have included in this paper recent studies on diluted magnetic semiconductors (DMSs), which are semiconductors where a small fraction of its atoms are magnetic. These materials are very promising for future applications in the area of spintronics. There are many formal similarities in the theoretical treatment of diluted magnetic semiconductors and the transition metal oxides mentioned above, justifying the inclusion of the former in this paper. Both classes of material present complex ground states, and this complexity leads to possible functionalities. Moreover, both systems require a careful treatment of electron interactions at the computational and theoretical level.

We will review recent results on these various correlated electron systems as studied with state-of-the-art computational techniques. For quantum dots, fundamental Hubbard models are considered. However, for CMR manganites and DMS materials more appropriate spinfermion models have been developed that, even though they could still be considered only as phenomenological models, include all the relevant interactions at a microscopic level. These models have been accurately studied using Monte Carlo algorithms combined with exact diagonalization of the fermionic sector. We will also discuss future directions of research in each of these areas.

\section{Recent studies of quantum dots and molecular conductors with computational techniques}

\subsection{Introduction}

The rapidly developing investigations in the area of nanometre-scale systems and their concomitant potential technological applications in real devices have induced considerable interest in the study of electron transport through single molecule conductors (MCs) and quantum dots (QDs). Due to the small size of these systems, Coulomb correlations are important. In addition, particularly in the case of single MCs, the electron-phonon coupling is also important, since molecules can change their shape and position relative to the leads as electrons enter or leave the system. This also has interesting effects on transport and other properties.

An important manifestation of electron correlations in nanostructures is the Coulomb blockade effect. The addition of an electron to a nanodevice is penalized by the Coulomb repulsion. Thus, the conductance is suppressed except for charge degeneracy points, where states with $N$ and $N+1$ electrons are degenerate, allowing for charge fluctuations. As a result, the conductance versus gate voltage curves present periodically spaced conductance peaks separated by low conductance valleys. This effect has been studied theoretically [1] and observed experimentally in both QDs [2] and single MCs [3, 4].

Another very interesting many-body effect is the Kondo effect. When an odd number of electrons occupy the device, conduction electrons in the leads screen their combined local magnetic moment. An overall singlet is formed. The antiferromagnetic correlations between the localized and conduction electrons lead to the formation of a resonance in the local 
density of states of the device. This resonance, the Kondo resonance, provides a channel for electron transport. The energy scale of this effect is defined as the Kondo temperature $T_{\mathrm{K}}$. Electron transport through the Kondo resonance was predicted theoretically [5-7], and has been observed experimentally in lithographic quantum dots [8], single molecules [3, 4, 9], carbon nanotubes [10], and other nanostructures.

More elaborate systems that show competitions between different correlation effects or more exotic Kondo effects have been studied. In the case of coupled QDs, the Kondo correlations between each dot and its neighbouring lead compete with the antiferromagnetic correlations between the two dots [11-13]. Ferromagnetic correlations have been predicted for coupled double-level QDs in the quarter-filling regime [14]. Integer-spin Kondo effect has been observed in multilevel QDs where the $T_{\mathrm{K}}$ is enhanced at the singlet-triplet degeneracy points [15]. Orbital Kondo effect, in addition to spin Kondo effect, has been observed in carbon nanotube based QDs. The conduction electrons in the leads screen both the orbital and spin degrees of freedom of the QD. When orbital and spin degeneracies are present simultaneously, a strongly enhanced Kondo effect obeying the $S U(4)$ symmetry is observed [16]. In addition to electronic correlation effects, vibrational effects in single MCs have received much theoretical attention [17-19] and have been observed in different experiments [20, 21].

\subsection{DMRG method to study conductances}

For a conceptual understanding of these complex systems, it is imperative to develop models and unbiased many-body methods that rely on a minimal number of assumptions in order to accurately handle both strong Coulombic and electron-phonon couplings. Several numerical techniques are being used to study electron transport in nanostructures (see [22] and references therein). The minimal model to describe QDs and single MCs is the one-level Anderson impurity model. This model can be written as $H=H_{\mathrm{D}}+H_{\text {leads }}+H_{\mathrm{D} \text {-leads }}$, where $H_{\mathrm{D}}$, the Hamiltonian of the QD (or molecule), is given by $H_{\mathrm{D}}=V_{\mathrm{g}} n_{\mathrm{d}}+U n_{\mathrm{d} \uparrow} n_{\mathrm{d} \downarrow}$. The first term represents the location of the energy level of the QD controlled by the gate voltage $V_{\mathrm{g}}$. The second term represents the Hubbard repulsion between electrons of opposite spins occupying the QD. $n_{\mathrm{d}}=n_{\mathrm{d} \uparrow}+n_{\mathrm{d} \downarrow}$ is the number of electrons at the dot. $H_{\text {leads }}$ is the Hamiltonian of the leads, modelled, in the results shown below, as ideal tight-binding chains. $H_{\mathrm{D} \text {-leads }}$ connects the dot to the leads. It can be written as $H_{\mathrm{D}-\text { leads }}=-t^{\prime} \sum_{\sigma}\left[c_{l \sigma}^{\dagger} d_{\sigma}+c_{r \sigma}^{\dagger} d_{\sigma}+\right.$ h.c.], where $t^{\prime}$ is the amplitude for the electronic hopping between the QD and the leads. $d_{\sigma}^{\dagger}$ creates an electron with spin $\sigma$ at the dot while $c_{l \sigma}^{\dagger}\left(c_{r \sigma}^{\dagger}\right)$ creates an electron with spin $\sigma$ at the last (first) site of the left (right) lead, if sites are numbered form left to right.

As an example of a powerful numerical technique used to study this type of system, we focus briefly on a recently proposed adaptive time-dependent DMRG-based technique to study nanotransport [22] (see also [23]). This technique has the advantages of being accurate, flexible enough to treat a wide spectrum of problems, and independent of any linear response assumptions. Thus, is can be used to study both equilibrium and non-equilibrium problems.

Figure 1 outlines the basic steps of the technique. In figure 1(a), a schematic representation of the geometry used is shown. The system in general consists of a relatively small interacting region connected to two leads. The leads are modelled by tight-binding Hamiltonians. The ground state at time zero is calculated at zero bias. Then, a finite bias $\Delta V$ is applied between the two leads and the wavefunction is evolved in time. The resulting current $J$ is measured as a function of time. The current typically shows a fast increase from zero followed by a steady-state plateau. The value of the current at the steady state is used to calculate the conductance. Figures $1(\mathrm{~b})$ and (c) show the exact results for $J(t) / \Delta V$ (in units of $e^{2} / h$ ) versus time (in units of $\hbar / t_{\text {leads }}$ where $t_{\text {leads }}$ is the hopping parameter in the leads) for the case of non- 
(a)

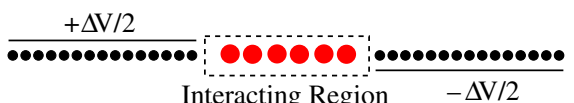

Interacting Region

$-\Delta \mathrm{V} / 2$
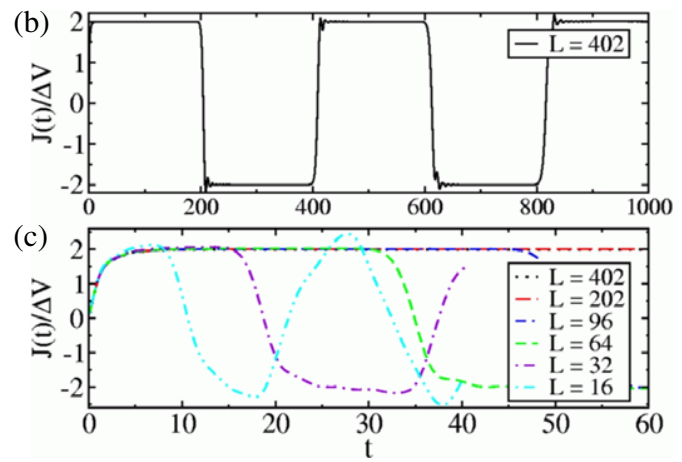

Figure 1. Adaptive time-dependent DMRG technique to study nanotransport. (a) Schematic representation of the geometry used. The leads are modelled by tight-binding Hamiltonians. The ground state at time zero is calculated at zero bias. Then, a finite bias $\Delta V$ is applied between the two leads and the resulting current is measured. (b) Exact results for $J(t) / \Delta V$ (in units of $e^{2} / h$ ) versus time (in units of $\hbar / t_{\text {leads }}$ ) for a cluster of length $L=402 . J(t) / \Delta V$ shows clear steadystate plateaus at $\pm 2 e^{2} / h$. The periodic changes in the current direction are caused by its reflection at the open boundaries of the cluster. (b) $J(t) / \Delta V$ obtained with decreasing $L$. The steady-state plateau is obtained even with $L=32$. The current is quasiperiodic with a period proportional to $L$ (from [22]).

interacting electrons and one quantum dot, obtained with clusters of different lengths $(L)$ and $\Delta V=0.001$. Figure $1(\mathrm{~b})$ shows $J(t) / \Delta V$ obtained with a large cluster $(L=401) . J(t) / \Delta V$ presents clear steady-state plateaus at $\pm 2 e^{2} / h$. The large-scale periodic changes in the current direction are caused by its reflection at the open boundaries of the cluster. Figure 1(c) shows $J(t) / \Delta V$ obtained with decreasing $L$. The steady-state plateau is obtained even with $L=32$. The current is quasiperiodic with a period proportional to $L$.

Figure 2 shows the conductance $G$ versus $V_{\mathrm{g}}$ for the 1QD case obtained using the procedure described above. Figure 2(a) shows $G$ deduced from the behavior of the current obtained with the DMRG method for a single 'non-interacting' QD, namely, one having $U=0$. It is expected that the maximum value of $G$ will be obtained when the level in the dot is aligned with the Fermi level of the leads, and this occurs in our case at $V_{\mathrm{g}}=0$. The DMRG results confirm this expectation. As $V_{\mathrm{g}}$ changes away from $0, G$ is expected to decrease symmetrically, and this is indeed shown in figure 2(a). In fact, the results at non-zero gate voltage are also in excellent quantitative agreement with the exact results. Figure 2(b) shows the results obtained for an intermediate value of $U$, namely $U=1$. These results are compared with the conductance obtained using the Friedel sum rule (FSR), $G=\frac{2 e^{2}}{h} \sin ^{2}\left(\frac{\pi}{2}\left\langle n_{d}\right\rangle\right)$. The shape of the curve is the expected one for the regime considered here: the intermediate value of $U$ does not locate our investigations deep in the Kondo regime, with sharply defined integer charge at the dot, but more into the so-called mixed-valence region. This can be deduced from the value of the dot charge versus $V_{\mathrm{g}}$, shown in figure 2(c). With increasing $U$ and/or decreasing $t^{\prime}$, sharper charge steps are formed, but the Kondo cloud size increases, thus requiring larger clusters to be used.

\subsection{Non-local spin control}

As an example of more elaborate nanosystems, we discuss briefly the results of recent important experiments involving non-local spin control [25]. We also discuss our numerical results 

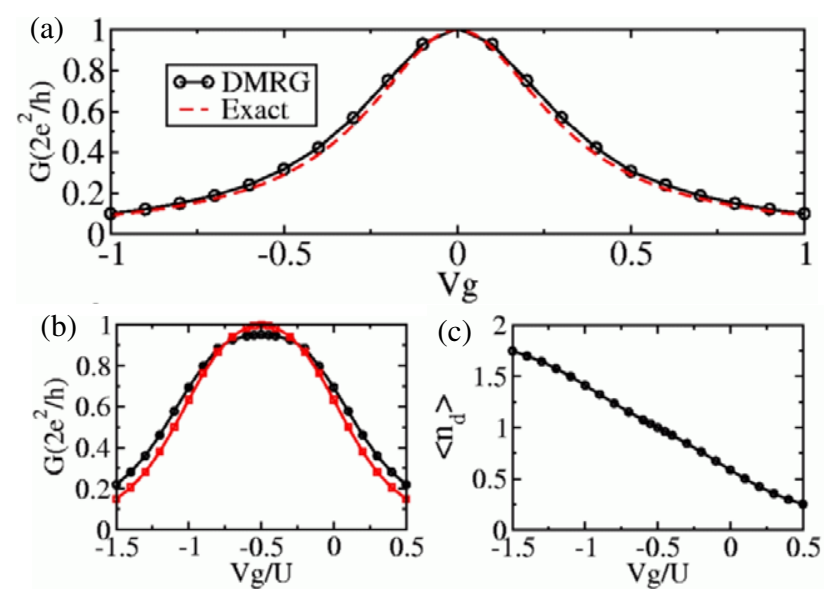

Figure 2. Adaptive time-dependent DMRG results in the case of one QD. (a) DMRG and exact results for $G$ versus $V_{\mathrm{g}}$ in the case of one non-interacting QD. The DMRG results are obtained with $L=64$ and number of states $M=300$. $G$ is obtained from the value of the steady-state current plateau. The exact results are for infinite leads. The plots show a resonant tunnelling peak with a full width at half maximum (FWHM) equal to $4 t^{\prime 2}$ at $V_{\mathrm{g}}=0$. (b) $G$ for one interacting QD. Circles show $G$ obtained by averaging the current over an interval of time corresponding to the steady state. Squares show $G$ obtained from $\left\langle n_{d}\right\rangle$ using the Friedel sum rule (FSR). $G$ has the shape of the expected Kondo or mixed-valence plateau centred at $V_{\mathrm{g}}=-U / 2$. This feature would become sharper by increasing $U$ and/or decreasing $t^{\prime}$. The results shown here were obtained using $U=1.0, t^{\prime}=0.4, L=128$, and $M=300$. (c) The dot occupation $\left\langle n_{d}\right\rangle$ for the same parameters (from [22]).

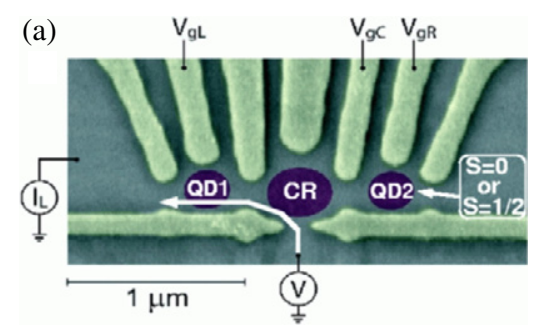

(b)

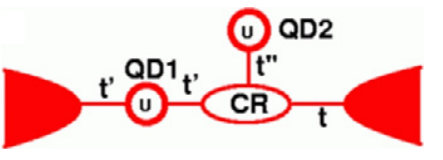

Figure 3. (a) Experimental setup used in the non-local spin control experiments. The system consists of two QDs (labelled QD1 and QD2) coupled through an open conducting central region (CR). The arrow indicates the direction in which bias is applied and conductance is measured. (b) Illustration of the model used in our numerical study. An Anderson impurity model is used to describe QD1 and QD2. QD2 is coupled to CR through the hopping parameter $t^{\prime \prime}$. Note that QD2 is side coupled to the direction in which the conductance is measured, thus a Fano antiresonance is quite natural in this system (from [24]).

simulating those experiments [24]. The experimental setup is shown in figure 3(a). The system consists of two similar QDs (labelled QD1 and QD2) coupled through an open conducting central region (CR). A finite bias is applied to QD1 as well as to the CR, while QD2 is kept at a constant gate potential. The differential conductance of QD1 is then measured for different 
charge states of QD2 and different values of its coupling to the CR. The main result was the suppression and splitting of the Kondo peak in QD1 by changing the occupancy of QD2 from an even to an odd number of electrons and by increasing its coupling to the CR. A RudermanKittel-Kasuya-Yosida (RKKY) interaction between the QDs was suggested as an explanation for the observed effects. Figure 3(b) shows the model used to simulate the experiment. The Anderson impurity Hamiltonian is used for both QDs. The calculations were carried out using the exact diagonalization plus Dyson equation technique [26]. Our numerical results nicely reproduced the experimental results. The system was also analysed using a simple circuit model, where the system is treated as a circuit of a serial QD (QD1) and a side-coupled QD (QD2) connected in series. The results of this simple analysis also reproduced the experimental results. The main conclusion of the numerical study was the following. The effects observed experimentally can also be explained as a result of the destructive interference of the two paths that an electron can take: a direct path through CR and QD1, and an indirect path through CR, QD2, CR and QD1. This so-called Fano antiresonance is quite natural in systems with side-coupled QDs.

\subsection{Molecular conductors}

As mentioned above, in the case of single MCs, in addition to the Coulomb interactions, the electron-phonon coupling is also important and can have interesting effects on transport properties. Let us discuss briefly the conductance of a molecule with centre of mass motion [17]. This system was studied numerically using the exact diagonalization plus Dyson equation technique. The molecule is allowed to oscillate between the two leads. It is clear that the molecule-leads tunnel barriers depend on the molecule displacement from its equilibrium position. The molecule is modelled using the Anderson-Holstein model. The Hamiltonian that connects the molecule to the leads can be written as $H_{\mathrm{M} \text {-leads }}=t^{\prime}\left[1-\alpha\left(a+a^{\dagger}\right)\right] \sum_{\sigma}\left(d_{\sigma}^{\dagger} c_{l 0 \sigma}+\right.$ h.c. $)+t^{\prime}\left[1+\alpha\left(a+a^{\dagger}\right)\right] \sum_{\sigma}\left(d_{\sigma}^{\dagger} c_{r 0 \sigma}+\right.$ h.c. $)$. In this model, $d_{\sigma}^{\dagger}$ creates an electron with spin $\sigma$ in the molecule, $t^{\prime}$ is the hopping parameter between the molecule and the leads, and $\alpha$ is a parameter that carries the dependence of $t^{\prime}$ on the molecule displacement from its equilibrium position $\hat{x}$ (note the opposite signs in this dependence for the two leads, caused by the molecules' centre of mass oscillations). This displacement can be written in terms of the phononic operators as $\hat{x}=\left(a+a^{\dagger}\right)$. The main result observed was the presence of a conductance dip at $V_{\mathrm{g}}=-U / 2$, for both the interacting and non-interacting electronic cases. In the following, we present an explanation of the conductance dip. The reasoning starts by noting that $H_{\mathrm{M} \text {-leads }}$ can be rewritten as a sum of two channels contributing to the overall molecule-leads connection. The first term, $t^{\prime} \alpha\left(a+a^{\dagger}\right) \sum_{\sigma}\left(d_{\sigma}^{\dagger} c_{r 0 \sigma}-d_{\sigma}^{\dagger} c_{l 0 \sigma}+\right.$ h.c. $)$, represents a phonon-assisted tunnelling channel, i.e. the electron absorbs (emits) a phonon upon entering the molecule and, then, emits (absorbs) a phonon upon leaving. The two channels were studied

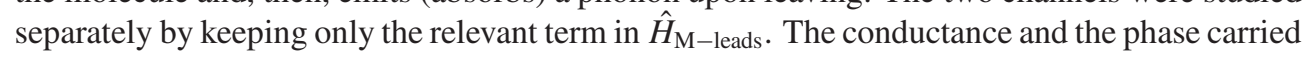
by each channel were calculated. It was found that, for $V_{g}=-U / 2$, the conductance of each of the channels is $2 e^{2} / h$ and the phase difference is $\pi$, leading to a perfect cancellation in the overall conductance. This interference effect is independent of the electron-electron interaction and, thus, the cancellation should still be present for $U=0$, as obtained numerically.

These systems provide a unique well-controlled and tunable playground, both for testing many-body theories and exploring new and exotic many-body effects. Some of these effects are very difficult or even impossible to realize in bulk system. In parallel with the huge experimental advances, reliable many-body, particularly numerical, techniques are essential to understand these increasingly interesting systems. This is an area of research with a bright future and surely with many interesting surprises waiting to be discovered. 


\section{Theoretical progress in colossal magnetoresistance}

\subsection{Introduction}

An important open problem in the area of transition metal oxides is the explanation of the colossal magnetoresistance (CMR) effect that appears in the Mn oxides that are widely referred to as manganites. These compounds present a rich phase diagram with a variety of competing states which are stabilized by changing the carrier concentration using a standard chemical doping process involving ions with different valences, or by varying the carrier bandwidth via isovalent doping. Among the low-temperature ground states in manganites there appears a ferromagnetic (FM) metallic phase and several antiferromagnetic/charge/orbital ordered insulating states. For the compounds with intermediate or small Curie temperatures, the experimentally obtained resistivity versus temperature curves present a sharp peak, which occurs precisely at the transition toward ferromagnetism. In the vicinity of this peak, the CMR effect is observed, which consists of enormous changes in the resistivity upon the introduction of relatively small magnetic fields. Technological applications of CMR compounds in the magnetic recording industry will still need a substantial increase of the currently available critical temperatures where the large magneto effects occur. However, the physics behind this remarkable CMR phenomenon defines a challenging basic-science problem that has attracted the attention of the condensed matter community.

The explanation of the CMR effect is one of the most important goals of theoretical investigations in the manganite context. It has been shown that the standard double exchange (DE) model was not sufficient to understand these materials [27]. In fact, a DE model cannot even produce an insulator at high temperatures, in the realistic regimes of electronic densities [28], and this pointed toward the importance of other couplings, such as electronphonon, for a proper description of these compounds. Progress was later made with the realization that manganite models have tendencies toward mixed phase regimes, typically involving metallic and insulating states in coexistence $[29,30]$. This discovery was possible only after the DE model and its close variations were studied with unbiased computational methods beyond mean-field approximations. Inhomogeneous states with a variety of length scales appear frequently in these studies and powerful computational tools are clearly needed to fully understand CMR materials [31]. The theoretical discovery of phase separation tendencies [32] was followed by an enormous experimental effort that confirmed the relevance of mixed states in most of the CMR compounds (for a review see [33]). Percolative pictures were envisioned to understand these materials. Model calculations by Mayr et al [34] and Burgy et al [35, 36], using simplified spin systems and random resistor networks, revealed a phenomenology very similar to that of real CMR materials in the regime of couplings and electronic densities where metallic and insulating states were in competition.

This initial effort using simple models was followed by calculations of resistivities in the more realistic, although still simplified, one-orbital model for manganites. Verges et al [37] numerically showed that an insulator can appear at intermediate and large temperatures if the electron-phonon coupling $\lambda$ is large enough. In this model, the two tendencies in competition at low temperatures are both ferromagnetic, and they only differ in the character of the charge distribution (uniform versus localized). The results considering only FM phases (metallic and insulating) cannot solve the entire CMR issue, since often the competition in experiments is between a ferromagnet against an antiferromagnetic/charge/orbital ordered state. However, those results are sufficiently interesting and challenging that they will be reviewed in detail here following closely [38]. Moreover, they could be of relevance to important Mn oxides such as $\mathrm{La}_{0.7} \mathrm{Ca}_{0.3} \mathrm{MnO}_{3}$, which at least naively seem well separated from charge ordered states in 
the phase diagrams. Recently, Kumar and Majumdar [39, 40] observed that the clean limit results of [37] are much enhanced by including on-site quenched disorder, together with a robust electron-phonon coupling.

Here we will review recent important progress related to the CMR problem. We will show that by increasing the electron-phonon coupling three regions are observed. First, for small electron-phonon coupling the system is metallic. As the electron-phonon coupling is increased past a critical value, a region in parameter space is found where the system has a metal insulator transition at finite temperature. If the electron-phonon coupling is too large the system becomes insulating for all temperatures. Even though the previous scenario is correct, the Hamiltonian parameters have to be tuned carefully to obtain a 'critical' region with a metal insulator transition at finite temperature. We show that the critical region is much enhanced when quenched disorder is added.

\subsection{Model and methods}

The Hamiltonian for the one-orbital model is

$$
\begin{aligned}
H_{1 b}=-t \sum_{\langle i j\rangle, \alpha}( & \left.c_{i, \alpha}^{\dagger} c_{j, \alpha}+\text { h.c. }\right)-J_{\mathrm{H}} \sum_{i, \alpha, \beta} c_{i, \alpha}^{\dagger} \vec{\sigma}_{\alpha, \beta} c_{i, \beta} \cdot \vec{S}_{i} \\
& -\lambda t \sum_{i, \gamma, \alpha}\left(u_{i,-\gamma}-u_{i, \gamma}\right) c_{i, \alpha}^{\dagger} c_{i, \alpha}+t \sum_{i, \gamma}\left(u_{i, \gamma}\right)^{2} \\
& +\sum_{i, \alpha}\left(\Delta_{i}-\mu\right) n_{i, \alpha}
\end{aligned}
$$

where $c_{i, \alpha}^{\dagger}$ creates an electron at site $i$ with spin $\alpha, \sigma_{\alpha, \beta}$ are the Pauli spin matrices, $\langle i j\rangle$ indicates summing over nearest-neighbour sites, and $t$ is the nearest-neighbour hopping amplitude for the movement of electrons ( $t$ also sets the energy unit, i.e. $t=1$ in all of the results below). The first and second terms are the standard for a double exchange model, with $\vec{S}_{i}$ being a classical localized spin that represents the $t_{2 \mathrm{~g}}$ degrees of freedom. The third term in the Hamiltonian accounts for the energy corresponding to the lattice-carrier interaction, with $\lambda$ being the strength of the electron-phonon coupling. $u_{i, \gamma}$ are the distortions (lattice displacements) of the oxygen atoms surrounding a Mn ion at site $i$. The index $\gamma$ in three dimensions (two dimensions) runs over three (two) directions $x, y$ and $z$ ( $x$ and $y$ ). The tendency toward increasing the magnitude of the lattice distortions is balanced by the fourth term in the Hamiltonian, which represents the stiffness of the Mn-O bonds. Since the study of quantum phonons in this context is not possible with currently available algorithms, the oxygen displacements are considered classical, an approximation widely used in studies of manganites [33]. Finally, the last term corresponds to the quenched disorder, which here it is introduced in the form of random site energies. $\Delta_{i}$ represents the strength of the disorder at a given site, and these numbers are chosen from a bimodal distribution of width $2 \Delta$ with mean 0 . The overall electronic density $n$ is controlled with the help of a chemical potential $\mu$ added to the last term in the Hamiltonian.

For manganites, $J_{H}$ is known to be large, and to simplify our study we consider the limit of $J_{H} \rightarrow \infty$, which has been shown to preserve the essential physics of manganites [33]. In this limit, the spin of the $\mathrm{e}_{\mathrm{g}}$-electron perfectly aligns along the localized $\mathrm{t}_{2 \mathrm{~g}}$-spin direction. For an infinite Hund coupling, the system is particle-hole symmetric with respect to density $n=0.5$. Thus, results at densities $n$ and $1-n$ are equivalent.

This Hamiltonian is solved using the standard exact diagonalization of the quadratic fermionic sector for a given spin background [33, 30]. The procedure, then, consists of integrating the $t_{2 g}$ spins (assumed to be classical) by means of a Monte Carlo algorithm. Details have been widely discussed in previous studies and they will not be repeated here [33, 30]. 


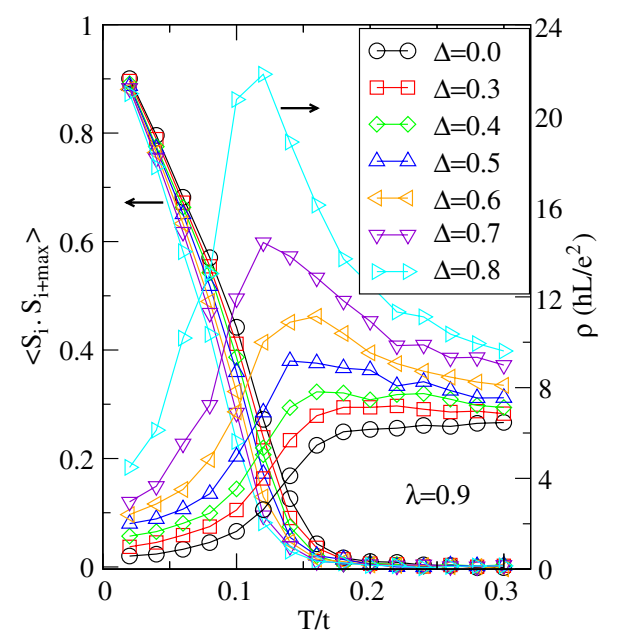

Figure 4. Monte Carlo results obtained using a $4 \times 4 \times 4$ lattice. Shown are the resistivity and spinspin correlations, the latter at the maximum allowed distance $(2 \sqrt{3})$, versus temperature, working at $\lambda=0.9, n=0.3$, and for the disorder strengths $\Delta$ indicated. The results shown are mainly for one configuration of quenched disorder, but as many as ten configurations were used in particular cases of temperatures and $\Delta$, and no substantial deviations were observed between disorder configurations (from [38]).

The resistivity $\rho$ has been calculated by taking the inverse of the mean conductivity $\sigma$, where the latter is related to the conductance $G$ by $G=\sigma L^{d-2}$, with $d$ being the dimension and $L$ the linear size of the lattice. The calculation of the conductance $G$ has been carried out following the approach extensively discussed before by Verges [41]. The use of the resistivity notation is to facilitate the interpretation of results and comparison with experiments, namely we do not claim to have observed ohmic behavior in our small system simulations. The units used for the resistivity are $\left[h / e^{2}\right]$ in two dimensions, and $\left[h / e^{2}\right] \times L$ in three dimensions. To restore the proper units to our results, the real lattice spacing of Mn oxides must be used.

\subsection{Resistivity}

The discussion of our computational results starts at the electronic density $n=0.3$ (equivalent to $n=0.7$, due to symmetry). Figure 4 is a typical example of the resistivity curves. Shown are both the spin-spin correlation at the maximum distance possible in the cluster under study and the resistivity, working at a fixed electron-phonon coupling $\lambda=0.9$, and varying the strength of the quenched disorder $\Delta$. In the clean limit, $\Delta=0$, there is a rapid change in resistivity near the transition to ferromagnetism. This is a typical pure double-exchange behavior: in the absence of a sufficiently strong $\lambda$, quenched disorder, or other couplings that may lead to competing states, then a metal is obtained at temperatures above the Curie temperature. As already clearly established in this field, pure double-exchange models are not enough to address the physics of the CMR materials. However, note the dramatic effect of quenched disorder on the resistivity, as shown in figure 4. Disorder can induce a peak in the resistivity that much resembles experimental results for typical CMR materials. Even for the small systems studied here, the ratio of resistivities between its maximum and minimum values is as large as $\sim 6$ for $\Delta=0.7$. Note the correlation between the peak location and the temperature where ferromagnetic order appears (signalled in our calculations by the value of the spin-spin correlation at the largest possible distance in the cluster under investigation). 


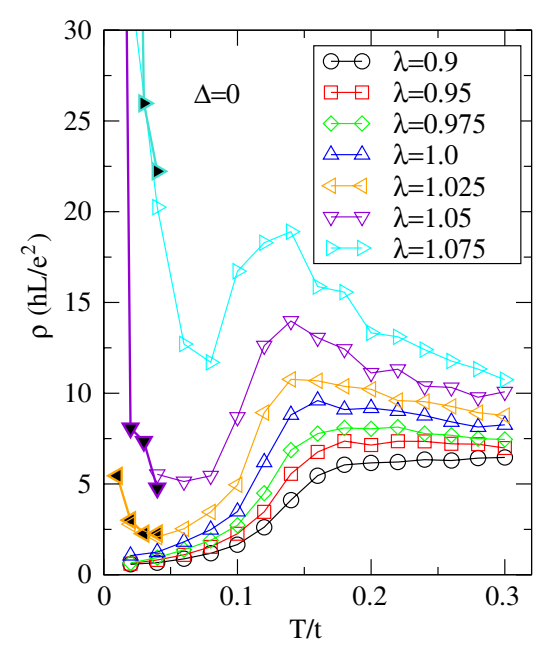

Figure 5. Influence of the electron-phonon coupling $\lambda$ on the resistivity curves in the clean limit $\Delta=0$, considering a $4 \times 4 \times 4$ lattice, and $n=0.3$ (from [38]).

Although a variety of previous theoretical and experimental investigations have convincingly shown the importance of quenched disorder in the CMR context, nevertheless it is interesting to observe that a resistivity peak can also be found by varying $\lambda$ even in the clean limit $\Delta=0$, as shown in figure 5 . There seems to exist a qualitative relation between increasing $\Delta$ at small $\lambda$ and simply increasing $\lambda$ at $\Delta=0$. However, there is an important difference between the two cases: observing the resistivity peak in the clean limit requires a fine tuning of $\lambda$. For a non-zero $\Delta$, the range of couplings with a resistivity peak is much wider (see below in the $n=0.1$ subsection for a more detailed discussion). Fine tuning is not compatible with the CMR effect since the phenomenon appears in a large number of manganese oxides, with a distribution of $\lambda$. For a discussion on the increase in the resistivity at very low temperature in figure 5 see [38].

The resistivity curves with peaks at intermediate temperatures (figures 4 and 5) resemble the experimental data corresponding to real manganites. Even if only qualitatively, the similarity of the Monte Carlo data in figures 4 and 5 with experimental observations is excellent.

\subsection{Magnetoresistance}

The study would not be complete without the investigation of the effect of a magnetic field. We will show that when a magnetic field is included, samples that present a peak in the resistivity also present a large magnetoresistance effect. In this subsection, $n=0.1$ will be considered (as already noted, $n=0.9$ is the same, via symmetry considerations for the model used). Several of the effects discussed at the realistic density $n=0.3$ in the previous section were found to be magnified by reducing the electronic density. Moreover, the lattice to be shown is now two dimensional, to illustrate the similarity systematically found between results in two and three dimensions.

The effect of magnetic fields at $n=0.1$ is very pronounced (see figure 6), resembling the magnitude of the CMR effect in real materials. The region in the vicinity of the resistivity peak is the most affected. The magnetoresistance ratios are as large as those reported in the real Mn oxides with the largest CMR effects. The trade-off is that the effect occurs only in a small 

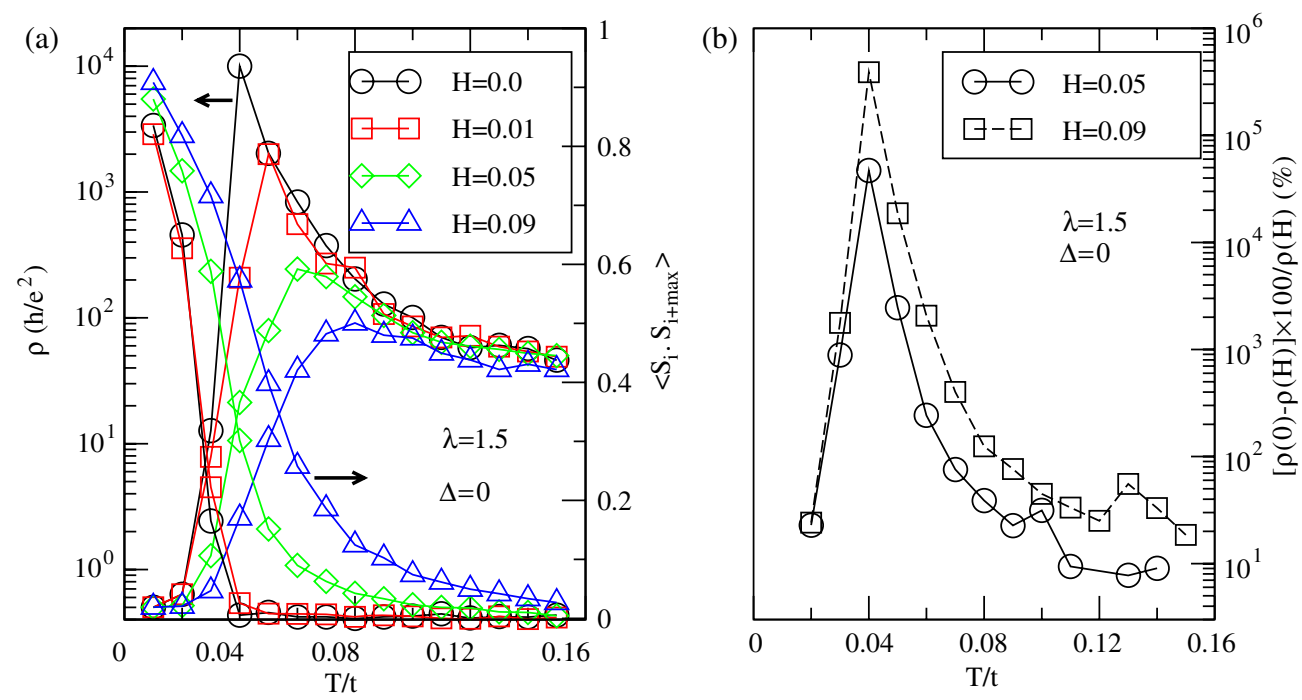

Figure 6. (a) Influence of magnetic fields on the resistivity curve and on the spin-spin correlation at the maximum allowed distance $(4 \sqrt{2})$ on an $8 \times 8$ lattice, in the clean limit $\Delta=0$. (b) Magnetoresistance ratios versus temperature, calculated for two representative magnetic fields. In both (a) and (b), $\lambda=1.5$ and $n=0.1$ (adapted from [38]).

window of $\lambda$, but this range, as well as the magnetoresistance value, can be further enlarged by adding quenched disorder.

The research effort discussed in previous publications by our group and others, and reviewed in this section, reached several goals. First, it confirmed the existence of a large peak in the resistivity versus temperature for the one-orbital model for manganites, including a robust electron-phonon coupling. This confirmation was obtained using unbiased techniques to estimate transport properties, and also with a Monte Carlo method that considers the complete Fermi sector without approximations. Second, a comprehensive analysis of the influence of couplings, quenched disorder strength, and electronic density was obtained. Although the last word has not still been said about the CMR phenomenon, considerable progress in its theoretical study has been reached in recent years. In particular, it is clear that the competition of two phases is crucial to have a colossal magnetoresistance.

\section{Diluted magnetic semiconductors: a dynamical mean-field study of a two-band model}

\subsection{Introduction}

Dynamical mean-field theory (DMFT) of strongly correlated electron systems replaces the full lattice of atoms and electrons with a single impurity atom imagined to exist in a bath of electrons $[42,43]$. While the theory maps the lattice model onto an impurity model, hence simplifying the spatial dependence of correlations among electrons, it still accounts fully for the their temporal dynamics, that is, for the local quantum fluctuations which are missing in an usual mean-field treatment [44]. Besides its conceptual value of providing a quantum analogue of the classical mean-field approach, the DMFT mapping of the lattice model onto an impurity model has important implications in approaching several complicated strongly correlated electron systems. Among such systems, the diluted magnetic semiconductors (DMSs), which are materials in which a small fraction $x(\approx 1-10 \%)$ of non-magnetic elements is replaced 
by magnetic impurities, attracted a substantial attention in the last decade due to their potential application in spintronic devices with new functionalities [45, 46]. Technologically, a semiconductor doped with magnetic atoms, usually $\mathrm{Mn}$, which combines both semiconductor properties and magnetism, was shown experimentally to exhibit ferromagnetic transition temperatures that led to expectations that such materials could be extensively applied in spintronics. Due to significant improvements in molecular beam epitaxy (MBE) techniques, a ferromagnetic transition temperature $\left(T_{\mathrm{C}}\right)$ of about $170 \mathrm{~K}$ has been recently recorded experimentally in Mn-doped GaAs [47]. In addition to its potential technological applications, the ferromagnetism of $\mathrm{Ga}_{1-x} \mathrm{Mn}_{x}$ As is a fundamental condensed matter problem as it represent a challenge to theory due to the combined presence of correlations and quenched disorder. The Mn dopants in GaAs serve the dual role of magnetic impurities that produce the local magnetic moments and of acceptors producing basically one hole per Mn atom. In turn, the density of charged carriers is itself a fraction $(\sim 10 \%)$ of the Mn concentration due to the strong localization at As antisite defects [48].

\subsection{Study of two bands}

Using dynamical mean-field theory (DMFT), in previous publications to be reviewed here we have studied the ferromagnetic transition temperature $\left(T_{c}\right)$ of a two-band model for diluted magnetic semiconductors (DMSs), varying the coupling constants, hopping parameters, and carrier densities. The DMFT treatment discussed here follows closely the results of [49]. We found that $T_{\mathrm{C}}$ is optimized at all fillings $p$ when both impurity bands (IBs) fully overlap in the same energy range, namely when the exchange couplings, as well as the bandwidths, are identical. The optimal $T_{\mathrm{C}}$ is found to be about twice as large as the maximum value obtained in the one-band model, showing the importance of multiband descriptions of DMSs at intermediate couplings. Within DMFT, pioneering calculations for two-band models based on double-exchange Hamiltonians already exist [50]. However, those calculations focused on special cases, while the present DMFT effort is more general, with two $s=1 / 2$ bands and arbitrary couplings, hoppings, and carrier densities. One of our main results is that $T_{\mathrm{C}}$ can be substantially raised by considering multiband systems since at intermediate couplings the maximal $T_{\mathrm{C}}$ at carrier filling $p \cong x$ is approximately twice as large as the highest $T_{\mathrm{C}}$ obtained in the single-band model at filling $p \cong x / 2$. The qualitative reason is that the IBs cooperate to raise $T_{\mathrm{C}}$ for values of the chemical potential $\mu$ where these bands partially or fully overlap [49].

It is known that in Mn-doped GaAs, the Mn ions substitute for $\mathrm{Ga}$ cations and contribute itinerant holes to the valence band. The $\mathrm{Mn}$ ions are in the $\mathrm{Mn}^{2+}$ state and have a half-filled $\mathrm{d}$ shell which acts as a $S=5 / 2$ local moment. Since the valence band of GaAs is p-like, a strong spin-orbit (SO) coupling between the angular momentum $\mathbf{l}=1$ of the p orbital and the hole spin degree of freedom $\mathbf{s}=1 / 2$ produces low- and high-energy bands with angular momentum $\mathbf{j}=1 / 2$ and $3 / 2$, respectively. A robust $\mathrm{SO}$ split between these bands $\left(\Delta_{\mathrm{SO}} \approx 340 \mathrm{meV}\right)$ causes the holes to populate the $\mathbf{j}=3 / 2$ state, which itself is split by the crystal field into an $m_{j}= \pm 3 / 2$ band with heavy holes and an $m_{j}= \pm 1 / 2$ band with light holes. Such a strong $\Delta_{\text {SO }}$ induces an anisotropic carrier-mediated interaction among the Mn ions that produces frustration in their ferromagnetic order [51], therefore reducing $T_{\mathrm{C}}$. While theoretical models might consider realistic effective masses for holes $\left(m_{\mathrm{h}} \approx 0.5 m_{\mathrm{e}}\right.$ and $m_{l} \approx 0.07 m_{\mathrm{e}}$ with a ratio $r=m_{l} / m_{\mathrm{h}} \approx 0.14 ; m_{\mathrm{e}}$ is the electron mass), studies have shown that $T_{\mathrm{C}}$ is maximized when giving similar masses to both heavy and light sectors [50]. The difference in band masses may induce magnetic frustration as the kinetic energy $\hat{K}=\sum_{\mathbf{k}} \hat{c}_{\mathbf{k}, \alpha}^{\dagger} \epsilon_{\mathbf{k}, \alpha \beta} \hat{c}_{\mathbf{k}, \beta}$ is only diagonalized when the angular momentum is quantized along the momentum direction [52]. Otherwise, with an arbitrary quantization direction at $r=1$ the magnetic frustration is absent. This argument 
may serve as an explanation for the recorded high values of $T_{\mathrm{C}}$ up to $250 \mathrm{~K}$ [53], since in deltadoped GaAs the reduction of the magnetic frustration might be associated with the restriction of $\mathrm{Mn}$ ions in two-dimensional planes, such a restriction allowing for the orientation of the Mn moments perpendicular to the plane.

Within DMFT, the weak-coupling quadratic dependence of $T_{\mathrm{C}}$ with the exchange $J$ is properly captured by using a one-band model for DMSs [54]. However, the predicted $T_{\mathrm{C}}$ versus $J$ dependence at large $J$ is almost a flat plateau, a result that has in turn been contradicted by the Monte Carlo (MC) simulations of finite clusters that correctly handle the random Mn distribution. While for $J$ comparable with the hopping $t$ both techniques reach similar conclusions, at large $J$ the $\mathrm{MC}$ techniques show the decay of $T_{\mathrm{C}}$ due to carrier localization $[55,56]$.

With two active bands that basically define its ferromagnetism, the Mn-doped GaAs becomes a subject of particular interest since the consideration of several active bands in real DMS materials beyond mean-field approximations is essential in understanding the ferromagnetism of DMSs and the occurrence of $T_{\mathrm{C}}$ close to room temperature [57, 58]. To account for both active bands, we introduced a simple two-band model based on the Hamiltonian:

$$
\mathcal{H}=-\sum_{l l^{\prime},\langle i j\rangle \alpha} t_{l l^{\prime}}\left(c_{l^{\prime}, j \alpha}^{\dagger} c_{l, i \alpha}+\text { H.c. }\right)-2 \sum_{l, I} J_{H, l} \mathbf{S}_{I} \cdot \mathbf{s}_{l, I},
$$

where $l, l^{\prime}(=1,2)$ are the band indices (not to be confused with angular momentum), $i, j$ label nearest-neighbour sites, $c_{l, i \alpha}$ creates a hole at site $i$ in the band $l, \mathbf{s}_{l, i}=c_{l, i \alpha}^{\dagger}\left(\sigma_{\alpha \beta} / 2\right) c_{l, i \beta}$ is the spin-operator of the mobile hole ( $\hat{\sigma}=$ Pauli vector), $\alpha$ and $\beta$ are spin indices, $J_{H, l}$ is the Hund coupling between the core spin and the electrons of band $l$, and $\mathbf{S}_{I}$ is the spin of the localized Mn ion at randomly selected sites $I$, assumed here classical $\left(\mathbf{S}_{i}=S \mathbf{m}_{i}\right.$, where $\mathbf{m}_{i}$ is a randomly orientated unit vector). While for $l=l^{\prime}$ we refer to $t_{l l}$ as the direct-orbital hopping ( $\equiv t_{l}$ ), for $l=l^{\prime}$ the off-diagonal hopping $t_{l l^{\prime}}$ is referred to as the interorbital hopping $\left(t_{l l^{\prime}}=t_{l^{\prime} l}^{*}\right)$ [59]. The two active bands couple when the carriers from both bands simultaneously scatter the same core spin and when the bands exchange carriers via the off-diagonal hopping. While the first coupling clearly causes an increase in $T_{\mathrm{C}}$ when the bands overlap within the same energy interval, namely when $J_{H, l}$ are closed, the exchange effect is expecting to produce its further boost [59].

\subsection{Results}

The DMFT technique applied to the Hamiltonian, equation (2), allows us to obtain the interacting density of states (DOS) and an implicit equation for $T_{\mathrm{C}}$. The $T_{\mathrm{C}}$ is then extracted from the structure of Matsubara frequencies numerically. For more details see [49]. The solutions of the DMFT equations for the interacting DOS depend crucially on the ratio $J_{l} / W_{l}$. The critical value for the formation of well-defined $\mathrm{IBs}^{6}$, corresponding to carrier spins locally parallel to Mn spins, is $J_{l} / W_{l} \sim 0.33$. In our study, the domain $J_{l} / W_{l}<0.33$ is referred usually to as 'weak coupling', while $0.33<\sim J_{l} / W_{l}<\sim 0.5$ is the 'intermediate coupling'. The most interesting physics is observed at the boundary between these two regimes, i.e. when the IBs are not completely separated from the valence bands.

In figure 7 , we show $T_{\mathrm{C}}$ versus $p$, for different ratios $J_{2} / J_{1}$ and at fixed $W_{2} / W_{1}=1$ and $J_{1} / W_{1}=0.5$, a situation corresponding to the existence of a well-defined $l=1 \mathrm{IB}$ (although

6 The term 'impurity band' is widely used in the DMS literature (see e.g. [61, 62]), although it should not be confused with the same term used in non-magnetic doped semiconductor literature. Perhaps 'exchange split valence band' could be a better terminology. 


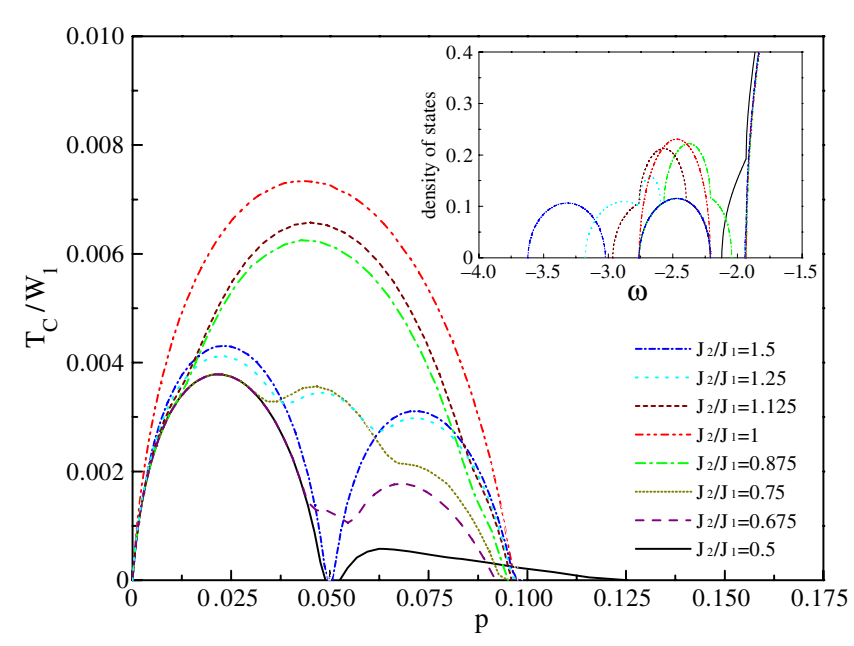

Figure 7. $T_{\mathrm{C}}$ versus the carrier concentration $p$, at various $J_{2} / J_{1}$. Here, $x=0.05, W_{1} / W_{2}=1$, and $J_{1} / W_{1}=0.5$. The inset shows the corresponding low-temperature interacting DOS (from [49]).

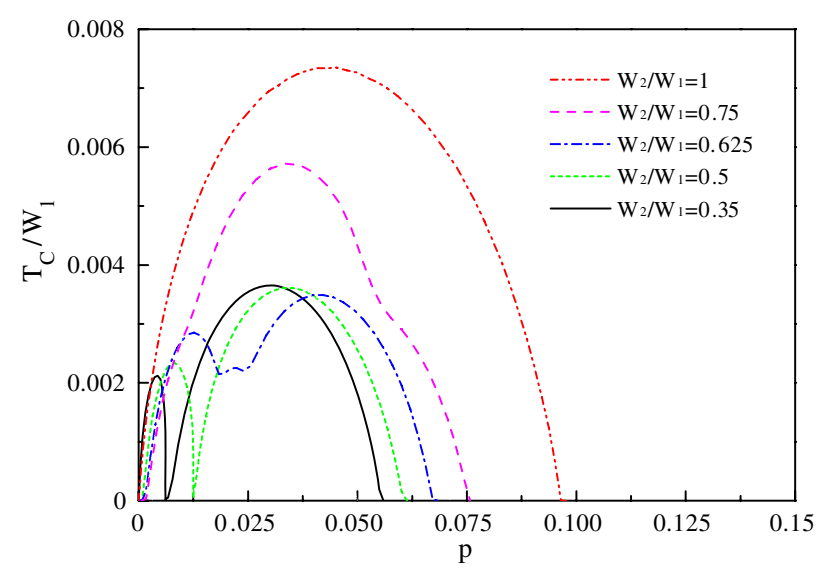

Figure 8. $T_{\mathrm{C}}$ versus $p$ at different ratios $W_{2} / W_{1}$, fixing $J_{1}=J_{2}=2$. Here $x=0.05$ (from [49]).

$p \leqslant x$ in real DMSs, the case $p>x$ is also studied for completeness, as done in [54]). The inset shows the total interacting DOS evolution. The IBs overlap if $\left|J_{2} / W_{2}-J_{1} / W_{1}\right|<0.5$. If the IBs do not overlap, then each one determines $T_{\mathrm{C}}$ separately, causing the double-peak structure observed for some $J_{2} / J_{1}$ ratios. The band with the largest $J_{l} / W_{l}$ is filled first, for smaller $\mu$. At all $p$, we found that $T_{\mathrm{C}}$ is maximum when $J_{2} / J_{1}=1$, namely when the IBs fully overlap. Once the bands decouple, the value for $T_{\mathrm{C}}$ matches one-band model results.

Let us consider now how changes in bandwidths influence $T_{\mathrm{C}}$. In figure 8 , we show $T_{\mathrm{C}}$ versus $p$ for different $W_{2} / W_{1}$, at fixed $J_{1} / W_{1}=0.5$ (intermediate coupling), and with $J_{2} / J_{1}=1 .^{7}$ At small $W_{2} / W_{1}$ the second IB will be located in a region of $\omega$ smaller (i.e. farther from the valence bands) than the energy interval occupied by the $l=1 \mathrm{IB}$. Hence, the $l=2$ IB will be the first to be filled. Decreasing $J_{2} / W_{2}$, the second band moves to the right on the $\omega$ axis, towards the location of the first band. While the bands are still separated, each gives its

7 The relative position of the bands is fixed by shifting the $l=2$ valence band numerically such that both valence bands start at the same energy, to mimic the degeneracy of the light and heavy bands of GaAs at the $\Gamma$ point. 


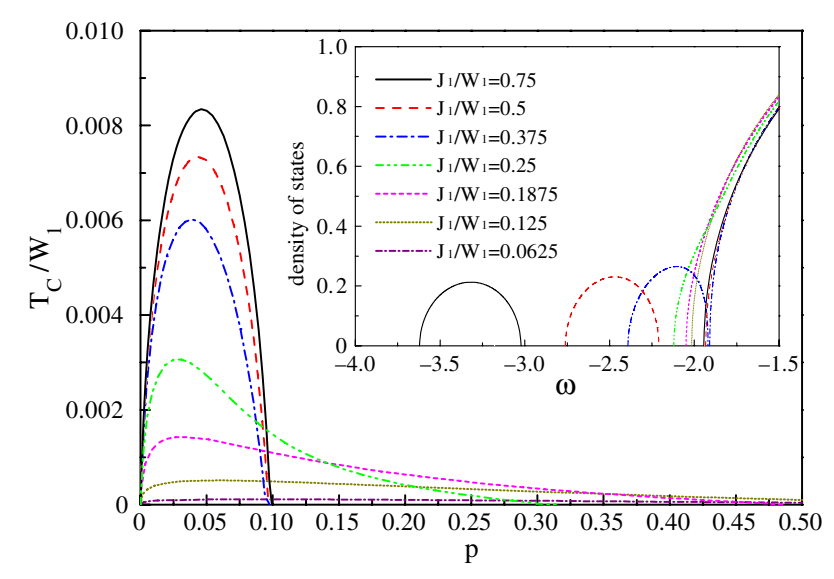

Figure 9. $T_{\mathrm{C}}$ versus $p$ at different ratios $J_{1} / W_{1}$, obtained using DMFT. The parameters $x, W_{1}, W_{2}$, and $J_{1}$ are as in figure 7. The inset shows the low-temperature interacting DOS (from [49]).

own contribution to $T_{\mathrm{C}}$. The curves with $W_{2} / W_{1}=0.35$ and 0.5 correspond to decoupled IBs, while those with $W_{2} / W_{1}=0.625$ and 0.75 correspond to partially overlapping bands. Again, $T_{\mathrm{C}}$ is maximized at all fillings when the bands fully overlap $\left(W_{2} / W_{1}=1\right)$, in good agreement with [50]. Although $W_{2} / W_{1}=1$ is not realistic in DMSs, we come to the conclusion that Mn-doped materials with a relatively small heavy-light mass ratio will favour a higher $T_{\mathrm{C}}$.

Now it is established that $T_{\mathrm{C}}$ is maximal for all $p$ when $J_{2} / J_{1}=1$ and $W_{2} / W_{1}=1$, let us analyse $T_{\mathrm{C}}$ versus $p$ when $J_{1} / W_{1}$ varies. The results for $T_{\mathrm{C}}$, and total interacting DOS, are in figure 9. At small coupling $J_{1} / W_{1} \ll 0.33, T_{\mathrm{C}}$ is small, flat, and much extended on the $p$ axis, results qualitatively similar to those found for one band [54]. However, at intermediate coupling, $T_{\mathrm{C}}$ is non-zero in the range from $p=0$ to $p=2 x$, adopting a parabolic form with the maximum at $p \cong x$, in contrast with the one-band model which gives a null $T_{\mathrm{C}}$ when $p \cong x$. The explanation is straightforward: at $p=x$ in the one-band model the IB is fully occupied, leading to a vanishing $T_{\mathrm{C}}$, but for the same $p$ in the two-band model both bands are half filled, which ultimately leads to the highest value for $T_{\mathrm{C}}$.

Concluding, the critical temperature $T_{\mathrm{C}}$ is maximized at intermediate couplings and for all carrier densities $p$ when $J_{1} / J_{2}=1$ and $W_{1} / W_{2}=1$. The maximum $T_{\mathrm{C}}$ is obtained at $p \cong x$, in contrast with the one-band model which shows a vanishing $T_{\mathrm{C}}$ at the same doping. In addition, $T_{\mathrm{C}}$ at filling $p \cong x / 2$ in the one-band case is smaller than with two bands by a factor $\sim 2$. In view of the simplicity of the main results, it is clear that adding an extra band to the calculations will only lead to a further increase in $T_{\mathrm{C}}$ when all the IBs overlap.

The approach described here is also quantitative. In fact, using realistic mean values specific to GaAs such as $p=0.005$, a bandwidth $\sim 10 \mathrm{eV}\left(t_{1} \approx 2.5 \mathrm{eV}\right), t_{2}=(1 / 9) t_{1}$, and assuming $J_{1} / t_{1}=1$ and $J_{1}=J_{2}$, we obtain $T_{\mathrm{C}} \approx 175 \mathrm{~K}$, i.e. within the experimental range. While this agreement with experiment [48] may be accidental, the trends are reliable and the result improves upon single-band estimations. Moreover, for optimal $t_{2} / t_{1}=1$ and $J_{1} / t_{1}=2$, the $T_{\mathrm{C}}$ raises to $\sim 340 \mathrm{~K}$, even at small $p=0.005$, setting the upper bound for DMSs under a two-band model description using a cubic lattice.

Our model presents several simplifications. For example, the Coulomb attraction by acceptors is not included, thereby neglecting the dominant energy of impurity states. The onsite Coulomb repulsion term $U \sum_{i} n_{i \uparrow} n_{i \downarrow}$, and the nearest-neighbour one $V \sum_{\langle i j\rangle} n_{i} n_{j}$ (where $n_{i}=n_{i \uparrow}+n_{i \downarrow}$ ), are not accounted for in the Hamiltonian (2). While the value of $T_{\mathrm{C}}$ is clearly 
affected by the Coulomb interactions, our model is still able to capture the main qualitative trends since the value of $V$, for instance, is comparable with the hopping and smaller than the coupling. On the other hand, the influence on $T_{\mathrm{C}}$ of the nearest-neighbour Coulomb repulsion may be suppressed by the exchange effect among bands that is expected to induce a boost of $T_{\mathrm{C}}$ once the off-diagonal hopping terms are accounted for.

\section{Conclusions}

The study presented in this brief review paper has covered several materials. In all of them, the effect of strong electronic correlations is important. For instance:

(i) Electron-electron and electron-phonon correlations play a crucial role in transport and other properties of nanostructures, as explained in section 2. Many of the observed effects usually occur only in these nanoscopic structures and not in the bulk. We have discussed new techniques, with particular emphasis on numerical methods, and have presented various results that can be obtained with these methods.

(ii) In section 3, a recent Monte Carlo study of the resistivity in a one-orbital spin-fermion (or double-exchange) model for manganites was briefly reviewed. A study of a two-orbital model for manganites has also been carried out (not discussed here but for details the reader can consult [38]). The overall conclusion is that its behavior is similar to that of the one-orbital model. Since these results are themselves also similar to experiments, our effort and those of other groups provide evidence that the theoretical studies that have focused on the regime of competition between a metal and an insulator are on the right track towards a full explanation of the CMR phenomenon. Both with one and two orbitals, quenched disorder is important in enlarging the magnitude of the effects and broadening its range in parameter space, thus avoiding the fine tuning of couplings needed in the clean limit.

The results reviewed in section 3 described mainly ferromagnetic phases both metallic and insulating. An area of study left for near future research is the important situation of non-ferromagnetic insulating phases competing with the ferromagnetic metallic regime. In fact, there has been some results concerning the effect of disorder on the insulating phases of manganites [60]. But more work is needed to address the effect of the superexchange coupling on the metal to insulator transition and on the formation of charge-ordered states.

(iii) Finally, the models and methods presented in section 4 can be used to search for diluted magnetic semiconductors with even higher $T_{\mathrm{C}}$ than currently known. Our results suggest that semiconductors with the heavy-to-light-hole mass ratio the closest to 1, such as AlAs, could have the highest $T_{\mathrm{C}}$ if the couplings $J$ could be tuned to its optimal value. The present effort paves the way toward future non-perturbative studies of DMS models using realistic zinc blende lattices, and points towards procedures to a further increase of the Curie temperature.

Overall, it can be concluded that the study of strongly correlated electronic systems continues providing interesting results, surprises, and soon may develop into an area of investigations with potential for real applications. Research in this field should continue to be strongly pursued by the condensed matter community.

\section{Acknowledgments}

GA is supported by the Eugene P Wigner Fellowship Program at Oak Ridge National Laboratory (ORNL). CS, AM, and ED are supported by the NSF grant DMR-0443144. This work is supported also in part by the LDRD program at ORNL. We acknowledge the National Center for Computational Sciences for providing most of the computational resources. ORNL 
is managed by UT-Battelle, LLC, for the US Department of Energy under Contract DE-AC0500OR22725.

\section{References}

[1] Meir Y, Wingreen N S and Lee P A 1991 Phys. Rev. Lett. 663048

[2] Meirav U, Kastner M A and Wind S J 1990 Phys. Rev. Lett. 65771

[3] Park J, Pasupathy A N, Goldsmith J I, Chang C, Yaish Y, Petta J R, Rinkoski M, Sentha J P, Abruna H D, McEuen P L and Ralph D C 2002 Nature 417722

[4] Liang W, Shores M P, Bockrath M, Long J R and Park H 2002 Nature 417725

[5] Glazman L I and Raikh M E 1988 JETP Lett. 47452

[6] Ng T K and Lee P A 1988 Phys. Rev. Lett. 611768

[7] Meir Y, Wingreen N S and Lee P A 1993 Phys. Rev. Lett. 702601

[8] Goldhaber-Gordon D, Shtrikman H, Mahalu D, Abusch-Magder U, Meirav U and Kastner M A 1998 Nature 391156

[9] Yu L H and Natelson D 2004 Nano Lett. 479

[10] Cobden D H, Nygard J and Lindelof P E 2000 Nature 408342

[11] Chang A M, Jeong H and Melloch M R 2001 Science 2932221

[12] Georges A and Meir Y 1999 Phys. Rev. Lett. 823508

[13] Büsser C A, Anda E V, Lima A L, Davidovich M A and Chiappe G 2000 Phys. Rev. B 629907

[14] Martins G B, Büsser C A, Al-Hassanieh K A, Moreo A and Dagotto E 2005 Phys. Rev. Lett. 94026804

[15] Sasaki S, De Franceschi S, Elzerman J M, van der Weil W G, Eto M, Tarucha S and Kouwenhoven L P 2000 Nature $\mathbf{4 0 5} 764$

[16] Jarillo-Herrero P, Kong J, van der Zant H S J, Dekker C, Kouwenhoven L P and De Franceschi S 2005 Nature 434488

[17] Al-Hassanieh K A, Büsser C A, Martins G B and Dagotto E 2005 Phys. Rev. Lett. 95256807

[18] Mitra A, Aleiner I and Millis A J 2004 Phys. Rev. B 69245302

[19] Cornaglia P S, Ness H and Grempel D R 2004 Phys. Rev. Lett. 93147201

[20] Park H, Park J, Lim A K L, Anderson E H, Alivisatos A P and McEuen P L 2000 Nature 40757

[21] Yu L H, Keane Z K, Ciszek J W, Cheng L, Stewart M P, Tour J M and Natelson D 2004 Phys. Rev. Lett. 93266802

[22] Al-Hassanieh K A, Feiguin A E, Riera J A, Büsser C A and Dagotto E 2006 Phys. Rev. B 73195304

[23] Schneider G and Schmitteckert P 2006 Preprint cond-mat/0601389

[24] Martins G B, Büsser C A, Al-Hassanieh K A, Anda E V, Moreo A and Dagotto E 2006 Phys. Rev. Lett. 96066802

[25] Craig N J, Taylor J M, Lester E A, Marcus C M, Hanson M P and Gossard A C 2004 Science 304565

[26] Büsser C A, Martins G B, Al-Hassanieh K A, Moreo A and Dagotto E 2004 Phys. Rev. B 70245303

[27] Millis A, Littlewood P and Shraiman B 1995 Phys. Rev. Lett. 745144

[28] Calderón M J, Vergés J A and Brey L 1999 Conductance as a function of temperature in the double-exchange model Phys. Rev. B 594170

[29] Moreo A, Yunoki S and Dagotto E 1999 Phase separation scenario for manganese oxides and related materials Science 2832034

[30] Dagotto E 2002 Nanoscale Phase Separation and Colossal Magnetoresistance (Berlin: Springer)

[31] Dagotto E 2005 Science 309258

[32] Yunoki S, Hu J, Malvezzi A, Moreo A, Furukawa N and Dagotto E 1998 Phys. Rev. Lett. 80845

[33] Dagotto E, Hotta T and Moreo A 2001 Colossal magnetoresistant materials: the key role of phase separation Phys. Rep. 3441

[34] Mayr M, Alvarez G and Dagotto E 2002 Global versus local ferromagnetism in a model for diluted magnetic semiconductors studied with Monte Carlo techniques Phys. Rev. B 65241202

[35] Burgy J, Mayr M, Martin-Mayor V, Moreo A and Dagotto E 2001 Colossal effects in transition metal oxides caused by intrinsic inhomogeneities Phys. Rev. Lett. 87277202

[36] Burgy J, Moreo A and Dagotto E 2004 Phys. Rev. Lett. 92097202

[37] Verges J A, Martin-Mayor V and Brey L 2002 Phys. Rev. Lett. 88136401

[38] Şen C, Alvarez G, Aliaga H and Dagotto E 2006 Phys. Rev. B 73224441

[39] Kumar S and Majumdar P 2005 Phys. Rev. Lett. 94136601

[40] Kumar S and Majumdar P 2006 Insulator-metal phase diagram of the optimally doped manganites from the disordered holstein-double exchange model Phys. Rev. Lett. 96016602

[41] Vergés J A 1999 Computational implementation of the Kubo formula for the static conductance: application to two-dimensional quantum dots Comput. Phys. Commun. 11871 
[42] Metzner W and Vollhardt D 1989 Phys. Rev. Lett. 32462

[43] Müller-Hartmann E 1989 Z. Phys. B 74507

[44] Georges A, Kotliar G, Krauth W and Rozenberg M J 1996 Rev. Mod. Phys. 6813

[45] Wolff S A 2001 Science 2941488

[46] Zutic I, Fabian J and Das Sarma S 2004 Spintronics: fundamentals and applications Rev. Mod. Phys. 76323

[47] Ohno H, Shen A, Matsukura F, Oiwa A, Endo A, Katsumoto S and Iye Y 1996 (Ga,Mn)As: a new diluted magnetic semiconductor based on GaAs Appl. Phys. Lett. 69363

[48] Ohno H 1998 Making nonmagnetic semiconductors ferromagnetic Science 281951

[49] Popescu F, Yildirim Y, Alvarez G, Moreo A and Dagotto E 2006 Phys. Rev. B 73075206

[50] Aryanpour K, Moreno J, Jarrell M and Fishman R S 2005 Phys. Rev. B 72045343

[51] Zaránd G and Jankó B 2002 Phys. Rev. Lett. 89047201

[52] Moreno J, Fishman R S and Jarrell M 2006 Phys. Rev. Lett. 96237204

[53] Nazmul A M, Amemiya T, Shuto Y, Sugahara S and Tanaka M 2005 Phys. Rev. Lett. 95017201

[54] Chattopadhyay A, Das Sarma S and Millis A J 2001 Transition temperature of ferromagnetic semiconductors: a dynamical mean field study Phys. Rev. Lett. 872272022

[55] Alvarez G, Mayr M and Dagotto E 2002 Phase diagram of a model for diluted magnetic semiconductors beyond mean-field approximations Phys. Rev. Lett. 89277202

[56] Alvarez G and Dagotto E 2003 Single-band model for diluted magnetic semiconductors: dynamical and transport properties and relevance of clustered states Phys. Rev. B 68045202

[57] Sinova J, Jungwirth T, Eric Yang S-R, Kucera J and MacDonald A H 2002 Infrared conductivity of metallic (III,Mn)V ferromagnets Phys. Rev. B 66041202

[58] Jungwirth T, König J, Sinova J, Kucera J and MacDonald A H 2002 Curie temperature trends in (III,Mn)V ferromagnetic semiconductors Phys. Rev. B 66012402

[59] Popescu F, Şen C and Dagotto E 2006 Phys. Rev. B 73 180404(R)

[60] Alvarez G, Aliaga H, Şen C and Dagotto E 2006 Phys. Rev. B 73224426

[61] Berciu M and Bhatt R N 2001 Phys. Rev. Lett. 87107203

[62] Okabayashi J, Kimura A, Rader O, Mizokawa T, Fujimori A, Hayashi T and Tanaka M 2001 Phys. Rev. B 64125304 